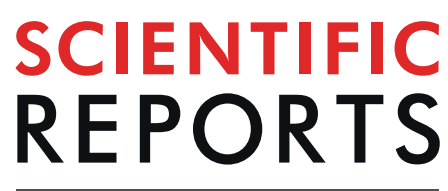

natureresearch

\title{
Hair cortisol concentrations are associated with hippocampal subregional volumes in children
}

\author{
Attila Keresztes ${ }^{1,2,3^{*}}$, Laurel Raffington ${ }^{2,4}$, Andrew R. Bender ${ }^{2,5}$, Katharina Bögl ${ }^{6}$, \\ Christine Heim ${ }^{7,8,10 *}$ \& Yee Lee Shing ${ }^{2,9,10}$
}

The human hippocampus, a brain structure crucial for memory across the lifespan, is highly sensitive to adverse life events. Stress exposures during childhood have been linked to altered hippocampal structure and memory performance in adulthood. Animal studies suggest that these differences are in part driven by aberrant glucocorticoid secretion during development, with strongest effects on the $C A 3$ region and the dentate gyrus (CA3-DG) of the hippocampus, alongside associated memory impairments. However, only few pediatric studies have examined glucocorticoid associations with hippocampal subfield volumes and their functional relevance. In 84 children (age range: 6-7 years), we assessed whether volumes of hippocampal subregions were related to cumulative glucocorticoid levels (hair cortisol), parenting stress, and performance on memory tasks known to engage the hippocampus. We found that higher hair cortisol levels were specifically related to lower CA3-DG volume. Parenting stress did not significantly correlate with hair cortisol, and there was no evidence to suggest that individual differences in hippocampal subregional volumes manifest in memory performance. Our results suggest that the CA3-DG may be the hippocampal region most closely associated with hair cortisol levels in childhood. Establishing causal pathways underlying this association and its relation to environmental stress and memory development necessitates longitudinal studies.

The hippocampus, a bilateral brain structure in the medial temporal lobes, is crucial for learning and memory in humans ${ }^{1}$. In particular, it supports recalling highly specific details of events in our lives ${ }^{2,3}$, constructing memories $^{4}$, associative or relational binding of information, and extracting knowledge from repeated experiences through generalization ${ }^{5,6}$. Importantly, it is also one of the most plastic ${ }^{7}$ brain structures, and is vulnerable to a wide range of influences across the lifespan ${ }^{8,9}$. The hippocampus is also implicated in the regulation of the hypothalamic-pituitary-adrenal (HPA) axis, and some evidence suggests the CA3 and the dentate gyrus subregions play a crucial role in stress adaptation ${ }^{10}$.

Renewed interest in the developmental plasticity of the hippocampus has been fueled by recent findings from high-resolution magnetic resonance imaging (MRI) research. Together, the evidence reveals a heterochronous pattern of protracted maturation across hippocampal subregions that continues well into adolescence ${ }^{11-15}$ and contributes to memory development ${ }^{16}$. This may be contrasted with prior suggestions that human hippocampal maturation occurrs by approximately 6 years of age ${ }^{17-19}$. Although the exact mechanisms underlying such plasticity are unclear, post-mortem studies in infants and very young children ${ }^{17,20}$, as well as data from various animal model $^{21,22}$ implicate synaptogenesis, dendritic branching, and neurogenesis in such developmental changes.

The hippocampus may also be highly vulnerable to adverse influences during development ${ }^{9,23,24}$. One primary source of such vulnerability is stress: various forms of chronic childhood stress including trauma, maltreatment, abuse, poverty, and stress-related mental diseases are associated with subsequently smaller hippocampal volume

\footnotetext{
${ }^{1}$ Brain Imaging Centre, Research Centre for Natural Sciences, Budapest, Hungary. ${ }^{2}$ Center for Lifespan Psychology, Max Planck Institute for Human Development, Berlin, Germany. ${ }^{3}$ Faculty of Education and Psychology, Eötvös Loránd University, Budapest, Hungary. ${ }^{4}$ Department of Psychology, University of Texas at Austin, Texas, USA. ${ }^{5}$ Departments of Epidemiology and Biostatistics \& Neurology and Ophthalmology, Michigan State University, Michigan, USA. ${ }^{6}$ Humboldt-Universität zu Berlin, Berlin, Germany. ${ }^{7}$ Charité - Universitätsmedizin Berlin, corporate member of Freie Universität Berlin, Humboldt-Universität zu Berlin, and Berlin Institute of Health, Institute of Medical Psychology, Berlin, Germany. ${ }^{8}$ Department of Biobehavioral Health, Pennsylvania State University, University Park, PA, USA. ${ }^{9}$ Institute of Psychology, Goethe University Frankfurt, Frankfurt am Main, Germany. ${ }^{10}$ These authors jointly supervised this work: Christine Heim and Yee Lee Shing. *email: keresztes.attila.akk@ttk.hu; christine.heim@charite.de
} 
in adulthood ${ }^{25-36}$. However, the retrospective nature of measuring childhood stress exposure and hippocampal volume in adulthood limits the validity of causal inferences that can be drawn from these extant findings. Investigations of stress-related effects on the hippocampus during childhood are scarce $\mathrm{e}^{31,37-46}$ and their results are mixed. Prior studies evaluating associations between hippocampal volume and history of stress-related psychiatric disorders, or neglect and maltreatment reported both positive $e^{38,46}$ and negative $e^{42,45,47,48}$ effects. Those few studies in healthy, non-institutionalized or maltreated children also reported both negative ${ }^{47}$ and positive ${ }^{49}$ associations between questionnaire-based measures of cumulative stress exposure and hippocampal volume. Moreover, the only two longitudinal studies reported either no change ${ }^{37}$ or a decrease in hippocampal volume over time in the stress group ${ }^{41}$. It is possible that reduced measurement reliability and specificity in those studies may have limited the valid measurement of hypothesized change.

Dysfunctions of the HPA axis regulating neuroendocrine responses to stress have been associated with alterations of hippocampal structure and function, although human evidence is limited ${ }^{33,50,51}$. Animal studies suggest the hippocampus is particularly sensitive to increased levels of specific stress hormones, such as mineralocorticoids and glucocorticoids due to its high density of receptors for these hormones ${ }^{52-55}$, and hippocampal vulnerability to glucocorticoids ${ }^{56}$. Studies with various mammal species, including primates, showed that moderate elevation of glucocorticoid concentrations in the hippocampus can lead to decreased neuronal survival rates following various adverse neurological events. Moreover, extremely high glucocorticoid concentrations can directly result in neuronal damage, as evidenced by reductions in cell numbers and dendritic arborization ${ }^{54,55,57}$, with glucocorticoid-induced dendritic atrophy in the CA3 observed in rodents ${ }^{58}$ suggested as a potential mechanism in humans ${ }^{34}$. These effects can, in addition, underlie observations of stress-induced decreases in neurogenesis ${ }^{59}$.

However, evidence on the relationships between stress, stress hormones, and maturation is mixed, suggesting a complex pattern of underlying associations ${ }^{60}$. For instance, stress does not always lead to changes in glucocorticoid levels ${ }^{34}$, and can instead - in some instances - be associated with reductions of glucocorticoid secretion in both animals and humans as measured in salivary and hair samples ${ }^{43,61-65}$. Importantly, this may also negatively affect neural maturation: low levels of glucocorticoids can negatively affect neurogenesis in interaction with $\mathrm{BDNF}^{66}$. Other studies report weak and inconsistent associations ${ }^{61,67,68}$ or null results ${ }^{67}$ on adversity and cortisol concentrations in children. A recent meta-analysis on the association between adversity and hair cortisol levels confirms that adversity can be associated with both higher and lower hair cortisol levels and that the small association is moderated by type and timing of adversity, and sample characteristics ${ }^{69}$. In addition, trauma may lead to increased intraindividual variance in glucocorticoid levels ${ }^{62}$. Thus, cortisol concentrations do not seem to function as straightforward biomarkers of chronic stress, although experimental studies clearly show that levels are stress-sensitive in children ${ }^{43,70}$.

In samples of human children that were not selected on the basis of institutionalization or trauma exposure, only three studies have evaluated the association between hippocampal volume and cortisol. Whereas two of these reported no association between salivary cortisol levels and total hippocampal volume ${ }^{43,71}$, Pagliaccio and colleagues ${ }^{49}$ found that a positive link between stressful life events and left hippocampal volume was mediated by salivary cortisol levels such that cortisol was negatively associated with both stressful life events and hippocampal volume.

Taken together, definitive conclusions from prior studies on stress-related differences in the hippocampus during childhood and its underlying mechanisms are limited. First, most studies investigated stress exposure retrospectively. Second, these studies have exclusively used salivary assays for measuring cortisol levels that are highly variable and influenced by various circadian and endocrine influences as well as many other factors (e.g., hydration, caloric intake). In contrast, newer techniques developed to extract glucocorticoids from human hair afford a more reliable estimation of glucocorticoid concentrations accumulated over longer periods of time $\mathrm{e}^{72,73}$. Third, associations of stress and cortisol do not appear to be uniform across the hippocampus ${ }^{71}$. In particular, the cornu ammonis regions and the dentate gyrus, the subregions with highest density of glucocorticoid receptors, may exhibit the largest negative cortisol effects in both animals and humans ${ }^{33,52,53,55,57}$. However, regionally specific effects within the human hippocampus have either been ignored or measured using methods with limited validity, largely due to methodological constraints imposed by standard resolution MRI.

Thus, in the present study, we investigated the association of cumulative levels of glucocorticoid concentrations, hippocampal subregional structure, and memory performance in an age-homogenous sample of 6-7-year old children. We assessed glucocorticoid concentrations via hair samples, and measured hippocampal subfield volumes using high-resolution MRI. We focused on this age range as it provides a window on development when the hippocampus is still undergoing changes, but when high-quality hippocampal structural images can already be acquired for reliable segmentation. Furthermore, we investigated whether self-reported parenting stress is associated with children's cumulative cortisol levels and hippocampal structure. Parenting stress has been linked to attenuated salivary diurnal cortisol secretion in their children, potentially reflecting the child's stress reaction to parenting stress ${ }^{64}$. Thus, assessing parents' perceived stress may allow an approximation of children's experienced stress levels, a construct difficult to measure reliably ${ }^{64}$. In addition, as the hippocampus has a key role in episodic memory, we evaluated children's performance on memory tasks known to depend on hippocampal function. Finally, we assessed children's performance on memory tasks, known to assess global (encoding spatial layouts ${ }^{74}$ ), and regionally specific (pattern separation in the dentate gyrus and CA $3^{3,75}$ ) hippocampal function.

Based on the available evidence from primates on the mechanisms of stress-induced cellular changes in the hippocampus, we hypothesized that glucocorticoid concentrations are either positively or negatively associated with hippocampal structure. Specifically, we expected the dentate gyrus and CA3 regions to be the most sensitive to glucocorticoid concentrations. In addition, we anticipated these associations to manifest in memory performance: positive associations between total hippocampal volume and spatial memory, and between regional volumes of the dentate gyrus and CA3 and pattern separation. 


\begin{abstract}
Methods and Materials
Participants. We recruited 147 children, aged six to seven years from six Berlin districts $\left(\mathrm{M}_{\text {age }}=2624\right.$ days [7.19 years], $\mathrm{SD}_{\text {age }}=167$ days; 67 girls) as part of a larger longitudinal study investigating SES-related stress effects on brain and cognition. Detailed description of the full sample, including risk of poverty, parental education and employment, child ancestry and bilingualism, as well as family status is given elsewhere ${ }^{43}$. Ethics approval was obtained from the 'Ethik-Kommission der Deutsche Gesellschaft für Psychologie' (Ethics Committee of the German Psychological Society). All research reported was performed in accordance with relevant guidelines and regulations. Informed consent was obtained from legal guardians of all participants: Parents provided written informed consent and children verbal assent. A subsample of randomly selected children had a magnetic resonance imaging (MRI) session that also incorporated behavioral tests on the mnemonic similarity task $(\mathrm{N}=84$; $\mathrm{M}_{\mathrm{age}}=2659$ days, $\mathrm{SD}_{\text {age }}=147$ days; 44 girls). Due to movement related artifacts, three participants had missing data for hippocampal subfields (one for all, one for left hemisphere only, and one for entorhinal cortex only). Due to various technical issues, mnemonic similarity task data was missing for 3 children, and due to a lack of consent to cut hair, hair cortisol data was missing for 12 participants with MRI data. Altogether, 64 children $\left(\mathrm{M}_{\text {age }}=2667\right.$ days, $\mathrm{SD}_{\text {age }}=142$ days; 31 girls) had complete data, however all analyses reported include all 84 children undergoing the MRI session (i.e., we did not exclude any participant with missing data).
\end{abstract}

Hippocampal subfield measures. We acquired high-resolution, partial field of view (FoV) volumes of the medial temporal lobe using a $\mathrm{T}_{2}$-weighted, proton density (PD)-weighted turbo spin echo sequence on a 3 T Siemens Magnetom TrioTim syngo MRI scanner with the following parameters: FoV: $206 \mathrm{~mm}$; repetition time (TR): $6,500 \mathrm{~ms}$; echo time (TE): $16 \mathrm{~ms}$; number of slices: 30 ; voxel size: $0.4 \mathrm{~mm} \times 0.4 \mathrm{~mm} \times 2.0 \mathrm{~mm}$, oblique to the coronal plane, perpendicular to the longitudinal axis of the right hippocampus to cover the full bilateral hippocampus.

To delineate regions within the hippocampus, we implemented a pipeline previously described in Bender et al. ${ }^{76}$ using the Automated Segmentation of Hippocampal Subfields (ASHS) software tool ${ }^{77}$ with a custom atlas also created using ASHS from manual segmentations with excellent reliability from earlier studies in our laboratory. This approach has been shown to be highly reliable and valid in identifying hippocampal subfield boundaries in a lifespan developmental sample, including 6-14 year-old children ${ }^{76}$. Instead of using a single-atlas with a one-size-fits-all approach as e.g., Freesurfer, ASHS uses multi-atlas method - integrating information from manual segmentations of multiple hippocampi - that provides greater anatomical precision on the individual level. Importantly, this ensures superior inherent validity of our subfield measurement approach as compared to other automated approached such as Freesurfer, which may produce less reliable estimates and generates less reliable labels for small anatomical regions.

We delineated three regions within the hippocampal body (Fig. 1) bilaterally - the subiculum, a region including Cornu ammoni (CA) regions 1 and 2 (CA1-2), and a region including CA3 and the dentate gyrus (CA3-DG). We chose not to divide CA3 from dentate gyrus, but rather collapsed them into one subfield (CA3-DG) because methods to reliably and validly separate the two structures on images acquired with 3 Tesla scanners - even if high-resolution - are yet to be established ${ }^{78}$. For the same reason, we collapsed CA1 with CA2 into one subfield (CA1-2), and also collapsed presubiculum, subiculum, and parasubiculum into one subfield (Subiculum). These regions were delineated only on the body because validity of delineation of these subfields in the head or the tail of the hippocampus is still debated ${ }^{78}$. In addition to subfields within the hippocampal body, we delineated the entorhinal cortex (Fig. 1) on 6 consecutive slices anterior to the hippocampal body, starting with the most anterior slice of the hippocampal body. The manual demarcation protocol we had used to create the custom atlas which was used to guide the automatic segmentations by ASHS in this study are presented in detail in section 2.3.2 and Fig. 1. of a previous publication ${ }^{76}$. To account for differences in ROI volumes due to differences in head size, we used the analysis of covariance approach ${ }^{79,80}$ to correct volumetric estimates of subfields for intracranial volume (ICV). The adjusted volumetric data is used for all ROIs throughout the present report. (See the Supplemental information for more details on hippocampal subfield volume assessment.)

Measurement of cortisol concentrations in hair samples. If children consented to hair sampling, a $\sim 1 \mathrm{~mm}$ thick strand of hair was taken from as close to the skull as possible to the tip of the hair. Hair samples were then taped on aluminium foil, wrapped and shipped to the Dresden LabService GmbH, Germany for analysis. Cortisol concentrations were determined using liquid chromatography tandem mass spectrometry ${ }^{72}$. The analysis was constrained to the first $3 \mathrm{~cm}$ long segment of the hair from the skull, which - based on an estimated average of $1 \mathrm{~cm} /$ month hair growth ${ }^{81}$ - contained hormones secreted over 3 months prior to taking the hair sample ${ }^{82}$. Cortisol concentrations $(\mathrm{pg} / \mathrm{mg})$ were log-transformed for parametric statistics.

Parenting stress measure. The Parenting Stress Inventor $y^{83}$ is a widely used questionnaire that assesses stress as a consequence of the parental role. Five subscales measure perceived stress due to child characteristics (Distractibility/Hyperactivity, Adaptability, Demandingness, Mood, Acceptability) and seven subscales measure parental characteristics and situational variables (Competence, Isolation, Attachment, Health, Role Restriction, Spouse/Parenting Partner Relationship, Depression). The validated German version of the questionnaire (Eltern-Belastungs-Inventar ${ }^{84}$ ) was completed by the parent who spent more time with the child and with the participating child in mind. Parents responded to 48 items on a 6-point scale ranging from strongly agree (0) to strongly disagree (5). The total score was divided by number of subscales (normally 12 subscales, but only 11 if the parent has no partner).

Memory measures. Spatial memory and pattern separation integrity were tested using a grid memory task and a mnemonic similarity task, respectively. Both were computerized tasks performed on desktop computers 
Sagittal view of $\mathrm{HC}$ body segmentation

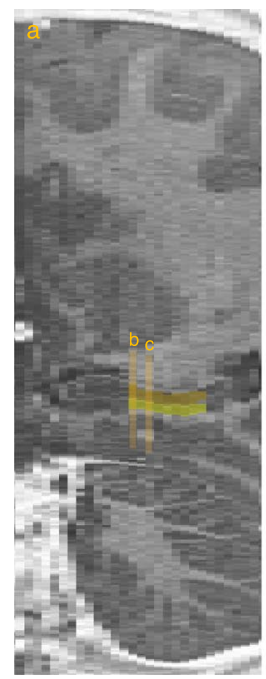

Example segmentations
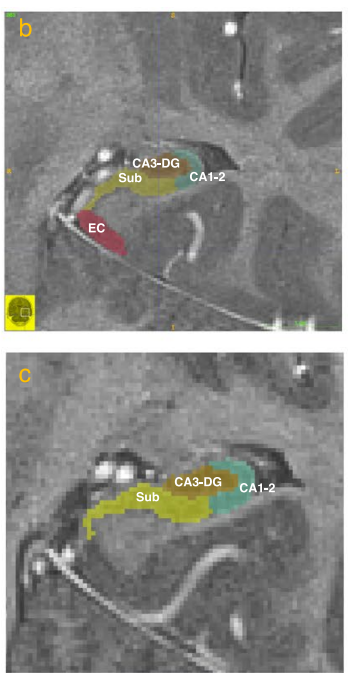

Most anterior slice of HC body

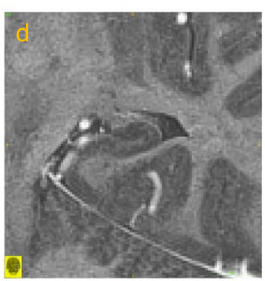

Most posterior slice of of HC head

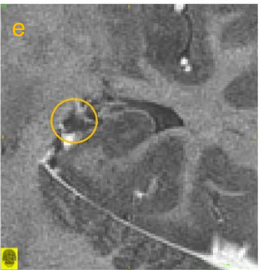

Most posterior slice of HC body

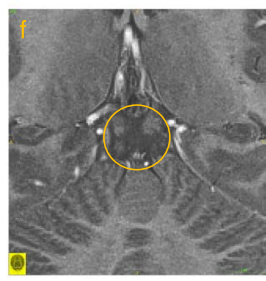

Most anterior slice of HC tail

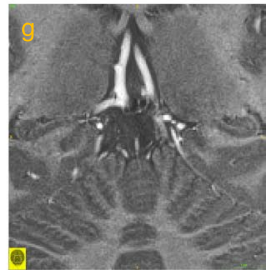

Figure 1. Illustration of Hippocampal Subfield Segmentations. (a) An example sagittal slice showing the extent of the hippocampal (HC) body segmentations were performed on. (b) The most anterior body slice with traces of all four regions overlaid. The four regions delineated comprised the Subiculum (Sub; with yellow color), a region including Cornu ammoni (CA) regions 1 and 2 (CA1-2; with turquoise color), a region including CA3 and the dentate gyrus (CA3-DG; with dark brown color), and the entorhinal cortex (EC; with red color). CA1-2, CA3-DG, and Subiculum were traced exclusively on HC body slices (from [D] to [F]), whereas EC was traced exclusively on (b) and 5 more slices anterior to it. (c) An additional example slice showing traces of CA1-2, CA3-DG, and Subiculum overlaid. (d) The most anterior HC body slice, defined as the first slice anterior to (e) which in turn was identified as the first slice on which the Uncus (circled) was clearly visible. (f) The most posterior slice of the HC body identified as the last slice on which the lamina quadrigemina (i.e., the inferior and superior colliculi; circled) are still clearly visible. (g) The first slice posterior to (f), where the lamina quadrigemina is not visible anymore. This slice was identified as belonging to the HC tail, and was not traced. Images created in ITK-SNAP ${ }^{109}$. See the Supplemental information for more details on hippocampal subfield volume assessment.

with standard 21.5-inch screens. The grid memory task was based on ${ }^{85}$, and assessed memory for items and item-location associations. In brief, participants encoded locations of 15 sequentially presented ( $3 \mathrm{~s}$ ) monochrome line drawings of everyday objects on gray-colored cells on a $6 \times 6$ grid. After a short delay, they performed a recognition task for the same pictures of studied objects randomly intermixed with 15 pictures of new objects. For each correctly recognized object, we asked them to point to the location of the given picture in the grid during encoding. As a measure of spatial memory, we used the percentage of correctly indicated locations for the 15 old items. For a detailed description of the task, $\operatorname{see}^{43}$.

The mnemonic similarity task was based ${ }^{86}{ }^{86}$, continuous recognition memory task assessing participants' ability to discriminate between memories of highly similar stimuli. Participants saw 162 pictures depicting everyday object, presented sequentially (trial duration: $4 \mathrm{~s}$, interstimulus interval: $0.5 \mathrm{~s}$ ). Critically, 48 pictures were repeated after a delay of either $2,6,10$, or 14 intervening trials. Twenty-four of the pictures were repeated exactly, whereas 24 other pictures were repeated with a slightly different lure picture of an identical object. For each trial the children's task was to identify pictures as "old" (exact repetition), "similar" (lure repetitions), or "new" (new items). Following ${ }^{86}$, we calculated a lure discrimination index as the proportion of "similar" responses to lure repetitions minus the proportion of "similar" responses to new items, that reflected participants' ability to discriminate between highly similar memories. Prior to the task, participants completed training (via PowerPoint slides) to use the three response options with unlimited response times, and then proceeded to a practice version with timings identical to the experiment.

Data conditioning and analysis. Data were screened for outliers, defined as having absolute values $>3$ SD from the mean. Outliers identified this way were further inspected and were subsequently excluded if obvious measurement errors were discovered. This resulted in the removal of one outlier ( $>10$ SD above mean; apparent measurement error) from the hair cortisol data. Hair cortisol estimation was not successful in five children, as their values fell below detection limit, potentially reflecting measurement errors such as hair being cut too far from the skull ${ }^{72}$. No other outliers were removed. Thus, with the additional missing data for 12 participants due to lack of consent to cut hair, hair cortisol data was available for 66 of the 84 children.

Structural equation modeling (SEM) was used to investigate the relationship between hippocampal subfield volumes, hair cortisol measure, and parenting stress. Importantly, by creating a latent factor for each hippocampal subfield using the left and right volume measures, SEM provides independent estimates for each subfield factor free of measurement error and hemisphere specific variation. All models were computed using maximum 


\begin{tabular}{|c|c|c|c|c|c|c|c|c|c|c|c|c|c|}
\hline & ERC left & $\begin{array}{l}\text { ERC } \\
\text { right }\end{array}$ & Sub left & Sub right & $\begin{array}{l}\text { CA1-2 } \\
\text { left }\end{array}$ & $\begin{array}{l}\text { CA1-2 } \\
\text { right }\end{array}$ & $\begin{array}{l}\text { CA3-DG } \\
\text { left }\end{array}$ & \begin{tabular}{|l|} 
CA3-DG \\
right
\end{tabular} & \begin{tabular}{|l|}
$\begin{array}{l}\text { Total HC } \\
\text { body }\end{array}$ \\
\end{tabular} & $\begin{array}{l}\text { Hair } \\
\text { cortisol }\end{array}$ & $\begin{array}{l}\text { Parenting } \\
\text { stress }\end{array}$ & $\begin{array}{l}\text { Grid } \\
\text { memory }\end{array}$ & LDI \\
\hline ERC right & $0.51 * * *$ & & & & & & & & & & & & \\
\hline Sub left & $0.37 * * *$ & $0.28^{* *}$ & & & & & & & & & & & \\
\hline Sub right & $0.27^{*}$ & $0.39 * * *$ & $0.6 * * *$ & & & & & & & & & & \\
\hline CA1-2 left & $0.34 * *$ & $0.4^{* * *}$ & $0.54 * * *$ & $0.3^{* *}$ & & & & & & & & & \\
\hline CA1-2 right & $0.3^{* *}$ & $0.49 * * *$ & $0.23^{*}$ & $0.52 * * *$ & $0.6 * * *$ & & & & & & & & \\
\hline CA3-DG left & $0.3 * *$ & $0.46^{* * *}$ & $0.55 * * *$ & $0.36 * * *$ & $0.81^{* * *}$ & $0.61 * * *$ & & & & & & & \\
\hline CA3-DG right & $0.25^{*}$ & $0.54 * * *$ & $0.25^{*}$ & $0.53 * * *$ & $0.54^{* * *}$ & $0.83 * * *$ & $0.68 * * *$ & & & & & & \\
\hline Total HC body & $0.4 * * *$ & $0.55^{* * *}$ & $0.71 * * *$ & $0.77 * * *$ & $0.76^{* * *}$ & $0.79 * * *$ & $0.83 * * *$ & $0.81^{* * * *}$ & & & & & \\
\hline Hair cortisol & -0.13 & -0.12 & -0.05 & -0.15 & -0.2 & -0.16 & -0.23 & $-0.29^{*}$ & -0.22 & & & & \\
\hline Parenting stress & $-0.25^{*}$ & -0.15 & -0.07 & -0.09 & 0.00 & -0.12 & -0.09 & -0.04 & -0.1 & 0.09 & & & \\
\hline Grid memory & -0.01 & -0.14 & -0.04 & 0.05 & -0.2 & -0.15 & $-0.25^{*}$ & -0.08 & -0.12 & -0.02 & 0.01 & & \\
\hline LDI & 0.06 & 0.16 & 0.14 & 0.1 & 0.14 & 0.03 & 0.16 & 0.05 & 0.13 & -0.01 & -0.03 & 0.15 & \\
\hline Age & -0.02 & 0.1 & -0.08 & 0.02 & 0.14 & $0.22^{*}$ & 0.1 & 0.2 & 0.11 & 0.04 & 0.06 & 0.03 & 0.17 \\
\hline
\end{tabular}

Table 1. Zero-order correlation matrix of all variables of interest. Note. ERC: entorhinal cortex, Sub: Subiculum, CA: Cornu Ammoni regions, DG: dentate gyrus, HC: hippocampus. Hair cortisol measures were $\log$ transformed. LDI: Lure discrimination index on the mnemonic similarity task. Age is age in days. ${ }^{*} \mathrm{p}<0.05$, $* * \mathrm{p}<0.01, * * * \mathrm{p}<0.001$, uncorrected for multiple comparisons.

likelihood estimation implemented in Onyx ${ }^{87}$ (version 1.0-1010). Standard goodness-of-fit indices, namely the root mean square error of approximation (RMSEA) and the comparative fit index (CFI), were used for evaluation of model fit. Models were considered a good fit with a RMSEA $<0.08$ and CFI $>0.95$ (e.g., Kline, 1998). The difference in $\chi^{2}$ fit statistics was used to compare nested models (Wald's test), with the degrees of freedom being the difference in the number of free parameters. The threshold for statistical significance was $p<0.05$. Both linear and quadratic associations were tested between variables of interest. In addition, to test for the robustness of results, we calculated $95 \%$ confidence intervals for all significant parameter estimates using 1000 bootstrapped resamples generated in lavaan ${ }^{88}$.

Age and Sex were included as covariates in further analyses, in light of earlier findings that have suggested an ongoing development of hippocampal structure in the age range examined ${ }^{12,15,89,90}$, and given known sex differences in HPA axis functioning e.g., ${ }^{43}$ as well as hippocampal subregions ${ }^{14,89,91,92}$. All indicator variables were converted to $\mathrm{z}$-scores.

\section{Results}

Table 1 provides the correlation matrix of all measures of interest plus Age. As can be seen, volumetric measures of hippocampal subfields were strongly intercorrelated, but subfield volumes and their sum - reflecting a total hippocampus (HC) body volume - were only weakly correlated with measures of hair cortisol concentrations, parenting stress, and memory performance, i.e., none of these weak correlations survive correction for multiple comparisons.

Associations between hippocampal subfield volumes and hair cortisol concentrations. First, a latent factor of each hippocampal subfield was specified using the left and right volume measures (standardized for each side) of the corresponding region as observed indicators. The two factor loadings of each subfield indicator were fixed to 1 . Latent factors had freely estimated covariances among each other, and residual variances of the indicators were not allowed to covary. The initial model showed a poor fit $\left(\chi^{2}=131.33, \mathrm{df}=18\right.$, $\mathrm{RMSEA}=0.208, \mathrm{CFI}=0.73$ ). It is plausible that there were significant covariances among the residual variances of the indicators that were not specified. Because of their close anatomical relationship, this was particularly likely for hippocampal subregions in the same hemisphere. Therefore, we allowed the residual variances of subiculum, CA1-2, and CA3-DG within each hemisphere to be correlated. This led to a model with a good fit $\left(\chi^{2}=14.66\right.$, $\mathrm{df}=12$, RMSEA $=0.039, \mathrm{CFI}=0.994$ ), which is the measurement model (see bottom two rows of Fig. 2 ) that we used for all subsequent steps.

Next, hair cortisol concentration was entered as an observed predictor variable of the latent subfields, while controlling for Age and Sex. In this model (see Fig. 2, and Table 2 for parameter estimates of variances, covariances, path values, and test statistics), hair cortisol concentrations negatively predicted the volume of dentate gyrus $(p=0.019)$. Of our covariate measures, individual differences in Age accounted for a significant portion of the variance in CA1-2 volume $(p=0.032)$, and showed a trend for a positive association with CA3-DG $(p=0.065)$, whereas Sex accounted for a significant portion of the variance in EC volume $(p=0.005)$, reflecting that girls had larger EC than boys. No other paths predicting brain volume were significant. Confidence intervals calculated from bootstrapped samples (see Table S3), provided support for the robustness of these associations. There were no non-linear associations between hair cortisol and hippocampal volumes (all standardized $\beta$ 's $<0.11$ and all $p$ 's $>0.13$ for quadratic terms). 


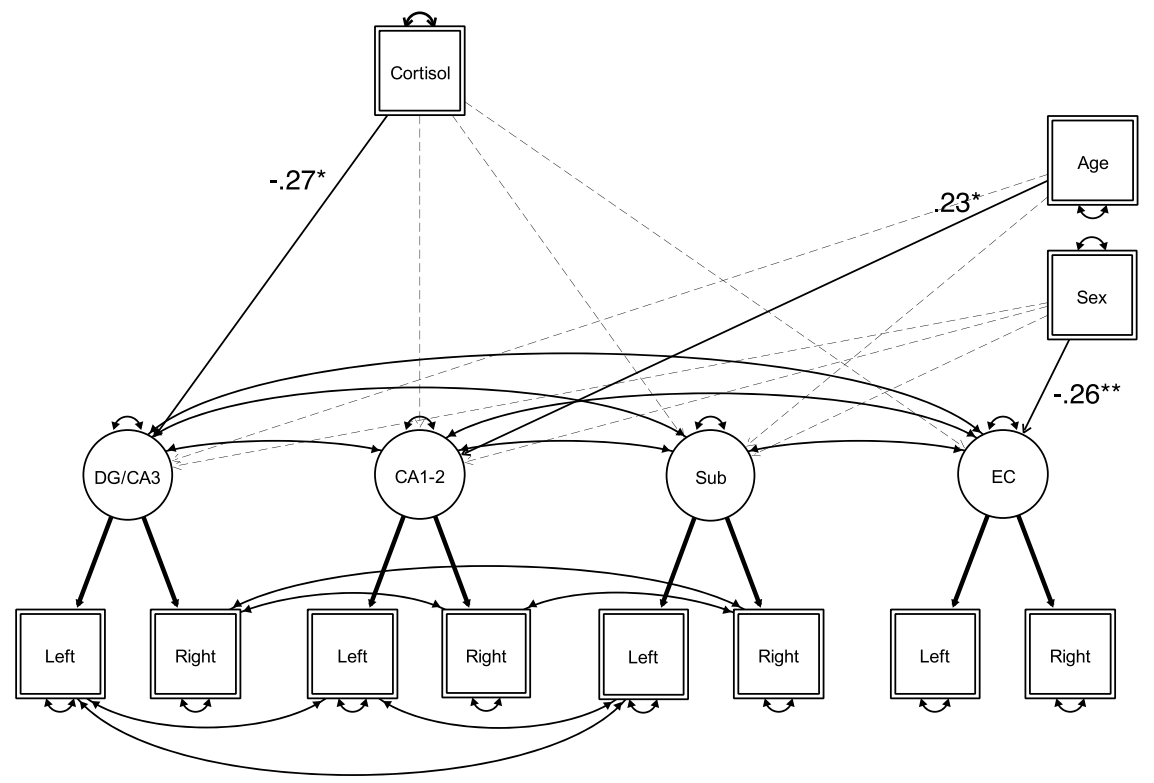

Figure 2. Schematic illustration of the hippocampal measurement model with regressions of hair cortisol concentrations (Cortisol), and covariates (Age and Sex) on hippocampal subfield volumes. DG/CA3: dentate gyrus-CA3, Sub: Subiculum: EC: Entorhinal cortex. Observed and latent variables are represented with rectangles and circles, respectively. Significant regression paths are shown as solid lines (with arrowhead) labeled with standardized parameter estimates. Non-significant paths are shown as dashed lines (with arrowhead). Estimated variances and covariances are also shown as solid lines. Thick solid lines represent path values fixed at 1 . All parameter estimates are shown separately in Table 2, for better readability of the figure.

Associations between parenting stress and hippocampal subfield volumes of children. Next, we explored associations between parental stress and children' hippocampal subfield volumes (see Figure S1, and Table S1). We specified parenting stress as a manifest variable with a regression paths to each of the four hippocampal subregion factors in the measurement model. In this model, parenting stress of parents was found to negatively predict the volume of entorhinal cortex $(p=0.018)$. Covariances with Age and Sex provided a similar pattern to the one observed in the hippocampal-hair cortisol model described above (see Table S1). No other paths predicting brain volume were significant. Again, bootstrapping replicated the results (see Table S3). There were no non-linear associations between parenting stress and hippocampal volumes (all standardized $\beta$ 's $<0.63$ and all $p$ 's $>0.15$ for quadratic terms).

Associations of hair cortisol concentrations, parenting stress, hippocampal subfield volumes and memory. A combined model with cortisol levels and parenting stress (see Figure S2 and Table S2) yielded an identical pattern of results with similar parameter estimates. Importantly, covariance between children's hair cortisol and parenting stress was positive, but not significant $(\mathrm{r}=0.08, S E=0.09, p=0.97)$. There were also no non-linear associations between children's hair cortisol, parenting stress and hippocampal volumes (all standardized $\beta$ 's $<0.11$ and all $p$ 's $>0.13$ for quadratic terms).

Next, spatial memory performance and lure discrimination index were entered in this combined model as manifest dependent variables regressed on the latent hippocampal subfield variables. Covariance between spatial memory and the lure discrimination index was positive, but not significantly different from zero $(r=0.18$, $S E=0.12, p=0.14)$. Hippocampal subfield volumes covaried both positively and negatively with memory performance, but never significantly different from zero (all standardized $\beta$ 's $<0.26$, all $p$ 's $>0.52$ ). There were also no non-linear associations between hippocampal volumes and memory (all standardized $\beta$ 's $<0.11$ and all $p$ 's $>0.12$ for quadratic terms).

Finally, in an additional analysis, we ruled out the potential confounding effect of participant's hair color on our main results. First, regressing hair cortisol on hair color revealed no significant association between the two $\left(\mathrm{R}^{2}=-0.014, \mathrm{~F}(3,87)=0.58, \mathrm{p}=0.63, \mathrm{~ns}\right.$. $)$. Second, when entering hair color as a dummy variable in a regression of right CA3-DG volume on hair cortisol, the significant association between hair cortisol and right CA3-DG volume remained significant $\left(\beta_{\text {standardized }}=0.30, \mathrm{p}=0.021\right)$.

\section{Discussion}

In this study, we examined whether 6-to-7-year-old children's hippocampal subregional volumes were associated with cumulative cortisol concentrations and parenting stress, as well as the functional relevance of differences in volume as indicated by memory performance. Based on the assumption that cumulative stress may be associated with altered cortisol concentrations, that in turn affect hippocampal volumes, and performance on hippocampal memory tests, we hypothesized a correlational relationship among these variables in our developmental sample. 


\begin{tabular}{|c|c|c|}
\hline \multirow[b]{2}{*}{ Model fit } & \multicolumn{2}{|c|}{$\chi^{2}=31.26, \mathrm{df}=38, \mathrm{RMSEA}=0, \mathrm{CFI}=1$} \\
\hline & $\begin{array}{l}\text { Parameter estimates } \\
\text { (Standard error) }\end{array}$ & $\Delta \chi^{2}(1)$ \\
\hline \multicolumn{3}{|l|}{ Latent variable variances } \\
\hline DG/CA3 & $0.58(0.12)$ & - \\
\hline CA1-2 & $0.52(0.12)$ & - \\
\hline Sub & $0.57(0.12)$ & - \\
\hline EC & $0.43(0.11)$ & - \\
\hline \multicolumn{3}{|l|}{ Latent variable covariances } \\
\hline DG/CA3 - CA1-2 & $0.49(0.11)$ & - \\
\hline DG/CA3 - Sub & $0.27(0.10)$ & - \\
\hline DG/CA3 - EC & $0.33(0.09)$ & - \\
\hline CA1-2-Sub & $0.24(0.10)$ & - \\
\hline CA1-2-EC & $0.32(0.08)$ & - \\
\hline Sub - EC & $0.30(0.09)$ & - \\
\hline \multicolumn{3}{|l|}{ Indicator covariances } \\
\hline \multicolumn{3}{|l|}{ Left hippocampus } \\
\hline DG/CA3 - CA1-2 & $0.29(0.09)$ & - \\
\hline DG/CA3 - Sub & $0.28(0.08)$ & - \\
\hline CA1-2-Sub & $0.30(0.08)$ & - \\
\hline \multicolumn{3}{|l|}{ Right hippocampus } \\
\hline DG/CA3 - CA1-2 & $0.20(0.07)$ & - \\
\hline DG/CA3 - Sub & $0.20(0.07)$ & - \\
\hline CA1-2-Sub & $0.23(0.08)$ & - \\
\hline \multicolumn{3}{|l|}{ Regression paths } \\
\hline Hair cortisol onto DG/CA3 & $-0.27(0.11)$ & $5.47^{*}$ \\
\hline Hair cortisol onto CA1-2 & $-0.18(0.11)$ & 2.63 \\
\hline Hair cortisol onto Sub & $-0.11(0.12)$ & 0.83 \\
\hline Hair cortisol onto EC & $-0.10(0.10)$ & 0.97 \\
\hline Age onto DG/CA3 & $0.20(0.11)$ & 3.41 \\
\hline Age onto CA1-2 & $0.23(0.10)$ & $4.58^{*}$ \\
\hline Age onto Sub & $-0.01(0.11)$ & 0.01 \\
\hline Age onto EC & $0.90(0.10)$ & 0.71 \\
\hline Sex onto DG/CA3 & $-0.08(0.10)$ & 0.70 \\
\hline Sex onto CA1-2 & $-0.13(0.10)$ & 1.93 \\
\hline Sex onto Sub & $-0.04(0.10)$ & 0.12 \\
\hline Sex onto EC & $-0.26(0.09)$ & $7.77 * *$ \\
\hline
\end{tabular}

Table 2. Parameter estimates of the hippocampal measurement model, with regressions of hair cortisol, and covariates (age, sex) on hippocampal subfield volumes. Note. Standardized parameter estimates shown with standard errors in parenthesis. Error variances are not shown. ${ }^{*} \mathrm{p}<0.05,{ }^{*} \mathrm{p}<0.01, * * * \mathrm{p}<0.001$.

Our most important finding is that hair cortisol concentrations over approximately three months preceding the study were negatively associated with the volume of the CA3-DG region of the hippocampus. Crucially, this may suggest that effects of cortisol accumulation may affect brain structure in healthy humans as early as 6 years of age. Such effects may have potential consequences for children in terms of learning and stress regulation. Given the cross-sectional nature of our results, it is also possible that children with smaller CA3-DG regions have subsequently higher cortisol secretion or a third variable causally accounts for their association. Importantly, this main finding replicates, with rigorous semi-automated hippocampal segmentation methods, recent findings of a concurrent study ${ }^{93}$. In contrast, previous studies exploring associations between cortisol and hippocampus in human children used different measures of salivary cortisol concentrations, and found mixed results e.g., 43 .

The specific association between cortisol and CA3-DG volume in children may reflect the increased vulnerability of this hippocampal region to high levels of cortisol concentrations via decreased survival rate of new neurons produced in the $\mathrm{DG}^{54,55,57,59}$, higher glucocorticoid-induced dendritic atrophy in the $\mathrm{CA}^{34}$, as well as a higher concentration of glucocorticoid receptors in the DG and CA regions that renders these regions more vulnerable to the diverse adverse effects of cortisol on hippocampal network integrity $33,52,53,55,57$.

We also found a negative association between parenting stress and volume in an adjacent neocortical region. We can speculate that this association may reflect a lack of resources that may support extra-hippocampal cortical development at this age by providing environmental stimulation. For instance, parental stress may be associated with limited resources to select environmentally stimulating experiences for the child. Alternatively, children with smaller EC volumes may evoke more parenting stress in their parents, or smaller EC volumes could reflect a heritable susceptibility to higher stress perception. However, why this may be specific to EC volumes - and not 
total HC - is unclear. Future studies should explore whether this association replicates in other samples, preferably in bidirectional longitudinal designs.

In this study associations between memory performance and volumetric measures of hippocampal subfields were not significantly different from zero, as we hypothesized. Thus, it is not possible to draw conclusions on whether the association between cortisol levels and hippocampus manifests in worse memory performance in children. Note that in another study using the same sample we found a trend for a negative association between total hippocampal volume (estimated with Freesurfer) and memory ${ }^{43}$. The lack of a robust association between memory and hippocampal structure in this sample seems to contradict earlier reports finding significant associations between performance on various memory tasks and total or region-specific hippocampal volumes $^{11-14,89,92,94,95}$. Most of these studies found a positive association between volume and memory (but see meta-analysis by Van Petten, 2004 that reported negative association). However, there is some evidence suggesting that the direction of the association may vary with age, across subregions, and along the longitudinal axis within the hippocampus ${ }^{89,90}$. These inconsistencies suggest a complicated relationship between memory and hippocampal volume that unfolds during development. For instance, Tamnes et al. ${ }^{92}$ found that cross-sectional associations (larger volume is better for retrieval) dissociate from longitudinal (decrease in volume is better for learning). This reflects a wider problem of false inferences from cross-sectional data with respect to true developmental patterns ${ }^{96}$, see also ${ }^{97}$. Another recent study ${ }^{89}$ found that the direction of the association between the volume of the CA1 region in the hippocampal head and memory shifts around the age of 6 from positive to negative. Thus, associations between memory and hippocampal volume may be better captured in larger cross-sectional samples spanning wider age ranges, or even better, by longitudinal sampling. Given the above considerations, the narrow age range of 6-7 years may have hindered the detection of any association of memory with hippocampal volume. This is especially so as pattern separation has been shown to develop beyond this age ${ }^{12,98}$. The possibility of an age range around or below the age of 6, during which hippocampal subfield volume is negatively associated with memory performance ${ }^{\text {cf. } 89}$ leads to the intriguing possibility that a smaller volume associated with higher cortisol levels may be beneficial for children of this age. This idea needs further exploration.

Another possibility is that the limited statistical power given the sample size and nature of the tasks in our study precluded detection of associations between memory and hippocampal volume. For instance, the continuous recognition task version of the mnemonic similarity task ${ }^{86}$ may put higher demands on working memory than the incidental task version ${ }^{99}$ used in our previous study ${ }^{12}$ where an association between age-related differences in hippocampal subfield volumes and memory was found. This is because in a continuous recognition task, participants are encouraged to monitor the stream of stimuli for reoccurring items and update their memories whenever an item appears again (as that item will no longer reoccur), which is very similar to the task demands of an $n$-back task. Thus, performance on this task may also heavily depend on prefrontal regions supporting working memory ${ }^{100}$, which may mask any association to the hippocampus present. Given the widely demonstrated association between hippocampal structure and memory performance, future studies may benefit from using child-adapted versions of tests of hippocampal function such as statistical inference ${ }^{14}$, holistic recognition ${ }^{101}$, and associative binding ${ }^{102}$.

We did not find a significant association between parenting stress (of the parents) and hair cortisol concentrations (in the children). This may be due to the fact that parenting stress is an indirect, and potentially unreliable approximation of children's stress levels, as well as a lack of statistical power, or a true lack of the association (either between parenting stress and children's stress, or children's stress and their hair cortisol levels). In addition, our parenting stress questionnaire did not specify that parents should fill it with respect to the preceding 3 months. Therefore, the time window covered by the parenting stress questionnaire did not precisely overlap with the three-month time window covered by the hair cortisol measurement. Self-reported parenting stress may also be a poor approximation of the parents' actual stress level ${ }^{103}$. However, a recent meta-analysis ${ }^{69}$ suggests that the association of adversity and hair cortisol levels is either positive and small in effects size or negative and moderate. Thus, our study may simply be underpowered to detect small-to-moderate associations. In addition, the non-significant association between hair cortisol in children and parenting stress of the parents may indicate that the parenting stress perceived by the parents does not translate directly into children's experienced stress ${ }^{\text {e.g., }} 64$. Alternatively, there may not be a reliable association of hair cortisol levels and stress exposure in children who were not institutionalized or maltreated ${ }^{\text {e.g., }}{ }^{67,68}$. Overall, this is in line with the notion that stress and cortisol have a complex, and yet poorly understood relationship ${ }^{60}$. Even in adults, and humans in general, within-person measurement of hair cortisol and its coupling with self-reported stress remains to be robustly corroborated (for evidence in primates, $\mathrm{see}^{73}$, for evidence in children $\mathrm{see}^{104}$, for a review see ${ }^{105}$ ). Our results do not provide evidence for the notion that the link between hair cortisol and hippocampal structure in children derives through a stress mechanism. Nevertheless, measurement of hair cortisol may be a promising tool for developmentalists investigating the relationship of cumulative cortisol concentrations (whether stress derived or not) and brain structure. This study provides initial evidence for a link between neural development and cumulative cortisol levels.

Finally, the negative association between CA3-DG and hair cortisol in children should be interpreted with caution until further variables beyond hair color can be ruled out as potential confounds. For instance, there is some evidence that hair washing, or repeated exposure to water (e.g., regular swimming) can leach cortisol out of primate $^{106}$ and human hair ${ }^{107}$, although human evidence is mixed and suggests that proximal segments of hair are not affected ${ }^{107,108}$. Importantly, a concurrent study similarly finding a negative CA3-DG - hair cortisol association, has successfully ruled out the modifying effect of hair washing ${ }^{93}$.

Based on the limitations touched upon in the above sections, future longitudinal studies may shed light on a true developmental lead-lag relationship between hair cortisol and hippocampal volume, or rather provide support for a common - perhaps genetic - mechanism. Longitudinal assessments are also needed to uncover a potential cascading effect of stress hormones. For instance, it is possible that effects on memory performance manifest only after longer periods of exposure to either too high or too low levels of cortisol concentrations. The 
development of valid assessment tools for measuring stress in children aged 6-7 years may further contribute to understanding the mechanisms underlying the observed association between hair cortisol and CA3-DG.

In sum, this study established a link between the accumulation of the stress hormone cortisol over a period of approximately three months and volume of the hippocampal region CA3-DG. It remains to be clarified to what extent our finding derives from environmental stress exposure, or mechanisms other than stress, such as genetic factors or toxin exposure, and whether the hair cortisol - CA3-DG association translates to effects on memory performance. We believe that uncovering these mechanisms could effectively guide efforts to understand developmental psychopathologies related to interactions of hormonal stress regulation in childhood and neurocognitive development.

Received: 25 October 2019; Accepted: 14 February 2020;

Published online: 17 March 2020

\section{References}

1. Scoville, W. B. \& Milner, B. Loss of recent memory after bilateral hippocampal lesions. J. Neurol. Neurosurg. Psychiatry 20, 11-21 (1957).

2. Tulving, E. Elements of episodic memory. (Clarendon, 1983).

3. Yassa, M. A. \& Stark, C. E. Pattern separation in the hippocampus. Trends Neurosci. 34, 515-525 (2011).

4. Hassabis, D. \& Maguire, E. A. Deconstructing episodic memory with construction. Trends Cognit. Sci. 11, 299-306 (2007).

5. Kumaran, D., Hassabis, D. \& McClelland, J. L. What learning systems do intelligent agents need? Complementary learning systems theory updated. Trends Cognit. Sci. 20, 512-534 (2016).

6. McClelland, J. L., McNaughton, B. L. \& O'Reilly, R. C. Why there are complementary learning systems in the hippocampus and neocortex: Insights from the successes and failures of connectionist models of learning and memory. Psychological Rev. 102, 419-457 (1995).

7. Shors, T. J. The adult brain makes new neurons, and effortful learning keeps them alive. Curr. Directions Psychological Sci. 23, 311-318 (2014)

8. Fjell, A. M., McEvoy, L., Holland, D., Dale, A. M. \& Walhovd, K. B. What is normal in normal aging? Effects of aging, amyloid and alzheimer's disease on the cerebral cortex and the hippocampus. Prog. Neurobiol. 117, 20-40 (2014).

9. Vargha-Khadem, F. et al. Differential effects of early hippocampal pathology on episodic and semantic memory. Sci. 277, 376-380 (1997).

10. McEwen, B. S. Physiology and Neurobiology of Stress and Adaptation: Central Role of the Brain. Physiological Rev. 87, 873-904 (2007).

11. Daugherty, A. M., Flinn, R. \& Ofen, N. Hippocampal CA3-dentate gyrus volume uniquely linked to improvement in associative memory from childhood to adulthood. NeuroImage 153, 75-85 (2017).

12. Keresztes, A. et al. Hippocampal maturity promotes memory distinctiveness in childhood and adolescence. PNAS 114, 9212-9217 (2017).

13. Lee, J. K., Ekstrom, A. D. \& Ghetti, S. Volume of hippocampal subfields and episodic memory in childhood and adolescence. NeuroImage 94, 162-171 (2014).

14. Schlichting, M. L., Guarino, K. F., Schapiro, A. C., Turk-Browne, N. B. \& Preston, A. R. Hippocampal structure predicts statistical learning and associative inference abilities during development. J. Cognit. Neurosci. 29, 37-51 (2017).

15. Tamnes, C. K., Bos, M. G. N., van de Kamp, F. C., Peters, S. \& Crone, E. A. Longitudinal development of hippocampal subregions from childhood to adulthood. Developmental Cognit. Neurosci. 30, 212-222 (2018).

16. Keresztes, A., Ngo, C. T., Lindenberger, U., Werkle-Bergner, M. \& Newcombe, N. S. Hippocampal maturation drives memory from generalization to specificity. Trends Cognit. Sci. 22, 676-686 (2018).

17. Frotscher, M. \& Seress, L. Morphological development of the hippocampus. in The hippocampus book 115-131 (Oxford University Press, 2007).

18. Gogtay, N. et al. Dynamic mapping of human cortical development during childhood through early adulthood. PNAS 101, 8174-8179 (2004).

19. Sowell, E. R. et al. Mapping cortical change across the human life span. Nat. Neurosci. 6, 309-315 (2003).

20. Ábrahám, H. et al. Myelination in the human hippocampal formation from midgestation to adulthood. Int. J. Developmental Neurosci. 28, 401-410 (2010).

21. Albani, S. H., McHail, D. G. \& Dumas, T. C. Developmental studies of the hippocampus and hippocampal-dependent behaviors: Insights from interdisciplinary studies and tips for new investigators. Neurosci. Biobehav. Rev. 43, 183-190 (2014).

22. Lavenex, P. \& Lavenex, P. Building hippocampal circuits to learn and remember: Insights into the development of human memory. Behavioural Brain Res. 254, 8-21 (2013).

23. de Haan, M., Mishkin, M., Baldeweg, T. \& Vargha-Khadem, F. Human memory development and its dysfunction after early hippocampal injury. Trends Neurosci. 29, 374-381 (2006).

24. Yu, Q. et al. Socioeconomic status and hippocampal volume in children and young adults. Developmental Sci. 21, e12561 (2018).

25. Buss, C. et al. Maternal care modulates the relationship between prenatal risk and hippocampal volume in women but not in men. J. Neurosci. 27, 2592-2595 (2007).

26. Dannlowski, U. et al. Limbic scars: long-term consequences of childhood maltreatment revealed by functional and structural magnetic resonance imaging. Biol. Psychiatry 71, 286-293 (2012).

27. Felmingham, K. et al. Duration of posttraumatic stress disorder predicts hippocampal grey matter loss. NeuroReport 20, 1402-1406 (2009).

28. Heim, C., Newport, D. J., Mletzko, T., Miller, A. H. \& Nemeroff, C. B. The link between childhood trauma and depression: Insights from HPA axis studies in humans. Psychoneuroendocrinology 33, 693-710 (2008).

29. Teicher, M. H., Anderson, C. M. \& Polcari, A. Childhood maltreatment is associated with reduced volume in the hippocampal subfields CA3, dentate gyrus, and subiculum. PNAS 109, E563-E572 (2012).

30. Vythilingam, M. et al. Childhood trauma associated with smaller hippocampal volume in women with major depression. AJP 159, 2072-2080 (2002)

31. Woon, F. L. \& Hedges, D. W. Hippocampal and amygdala volumes in children and adults with childhood maltreatment-related posttraumatic stress disorder: A meta-analysis. Hippocampus 18, 729-736 (2008).

32. Bremner, J. D. et al. Magnetic resonance imaging-based measurement of hippocampal volume in posttraumatic stress disorder related to childhood physical and sexual abuse-a preliminary report. Biol. psychiatry 41, 23-32 (1997).

33. Frodl, T. \& O'Keane, V. How does the brain deal with cumulative stress? A review with focus on developmental stress, HPA axis function and hippocampal structure in humans. Neurobiol. Dis. 52, 24-37 (2013).

34. Lupien, S. J., McEwen, B. S., Gunnar, M. R. \& Heim, C. Effects of stress throughout the lifespan on the brain, behaviour and cognition. Nat. Rev. Neurosci. 10, 434-445 (2009). 
35. Pattwell, S. S. \& Bath, K. G. Emotional learning, stress, and development: An ever-changing landscape shaped by early-life experience. Neurobiol. Learn. Mem. 143, 36-48 (2017).

36. Tottenham, N. \& Sheridan, M. A. A review of adversity, the amygdala and the hippocampus: a consideration of developmental timing. Front Hum Neurosci 3, (2010).

37. Bellis, M. D. D., Hall, J., Boring, A. M., Frustaci, K. \& Moritz, G. A pilot longitudinal study of hippocampal volumes in pediatric maltreatment-related posttraumatic stress disorder. Biol. Psychiatry 50, 305-309 (2001).

38. Bellis, M. D. D. et al. Developmental traumatology part II: brain development. Biol. Psychiatry 45, 1271-1284 (1999).

39. Bellis, M. D. D. et al. Brain structures in pediatric maltreatment-related posttraumatic stress disorder: a sociodemographically matched study. Biol. Psychiatry 52, 1066-1078 (2002).

40. Carrion, V. G. et al. Attenuation of frontal asymmetry in pediatric posttraumatic stress disorder. Biol. Psychiatry 50, $943-951$ (2001).

41. Carrion, V. G., Weems, C. F. \& Reiss, A. L. Stress predicts brain changes in Children: A pilot longitudinal study on youth stress, posttraumatic stress disorder, and the hippocampus. Pediatrics 119, 509-516 (2007).

42. Dahmen, B. et al. Effects of early-life adversity on hippocampal structures and associated HPA axis functions. DNE 40, 13-22 (2018).

43. Raffington, L. et al. Blunted cortisol stress reactivity in low-income children relates to lower memory function. Psychoneuroendocrinology 90, 110-121 (2018).

44. Rao, H. et al. Early parental care is important for hippocampal maturation: Evidence from brain morphology in humans. NeuroImage 49, 1144-1150 (2010).

45. Rao, U. et al. Hippocampal changes associated with early-life adversity andvulnerability to depression. Biol. Psychiatry 67, 357-364 (2010).

46. Tupler, L. A. \& De Bellis, M. D. Segmented hippocampal volume in children and adolescents with posttraumatic stress disorder. Biol. Psychiatry 59, 523-529 (2006).

47. Hanson, J. L. et al. Behavior problems after early life stress: contributions of the hippocampus and amygdala. Biol. Psychiatry 77, 314-323 (2015).

48. Lambert, H. K. et al. Hippocampal contribution to context encoding across development Is disrupted following early-life adversity. J. Neurosci. 37, 1925-1934 (2017).

49. Pagliaccio, D. et al. Stress-system genes and life stress predict cortisol levels and amygdala and hippocampal volumes in children. Neuropsychopharmacology 39, 1245-1253 (2014).

50. McEwen, B. S. Stress and Hippocampal Plasticity. Annu. Rev. Neurosci. 22, 105-122 (1999).

51. Schoenfeld, T. J. \& Gould, E. Stress, stress hormones, and adult neurogenesis. Exp. Neurol. 233, 12-21 (2012).

52. Patel, P. D. et al. Glucocorticoid and mineralocorticoid receptor mRNA expression in squirrel monkey brain. J. Psychiatr. Res. 34, 383-392 (2000).

53. Reul, J. \& De Kloet, E. R. Anatomical resolution of two types of corticosterone receptor sites in rat brain with in vitro autoradiography and computerized image analysis. J. steroid Biochem. 24, 269-272 (1986).

54. Sapolsky, R. M., Uno, H., Rebert, C. S. \& Finch, C. E. Hippocampal damage associated with prolonged glucocorticoid exposure in primates. J. Neurosci. 10, 2897-2902 (1990).

55. Uno, H., Eisele, S., Baker, E., DeJesus, O. \& Holden, J. Neurotoxicity of glucocorticoids in the primate brain. Hormones Behav. 28, 336-348 (1994).

56. Conrad, C. D. Chronic Stress-induced Hippocampal Vulnerability: The Glucocorticoid Vulnerability Hypothesis. Rev. Neurosci. 19, 395-412 (2008)

57. Uno, H. et al. Brain damage induced by prenatal exposure to dexamethasone in fetal rhesus macaques. I. Hippocampus. Brain Res. Dev. Brain Res. 53, 157-167 (1990).

58. Magarin os, A. M. \& McEwen, B. S. Stress-induced atrophy of apical dendrites of hippocampal CA3c neurons: Involvement of glucocorticoid secretion and excitatory amino acid receptors. Neurosci. 69, $89-98$ (1995).

59. Hueston, C. M., Cryan, J. F. \& Nolan, Y. M. Stress and adolescent hippocampal neurogenesis: diet and exercise as cognitive modulators. Transl. Psychiatry 7, e1081 (2017).

60. McEwen, B. S. What Is the Confusion With Cortisol? Chronic Stress. 3, 2470547019833647 (2019).

61. Bunea, I. M., Szentágotai-Tătar, A. \& Miu, A. C. Early-life adversity and cortisol response to social stress: a meta-analysis. Transl. Psychiatry 7, 1274 (2017).

62. Dajani, R., Hadfield, K., van Uum, S., Greff, M. \& Panter-Brick, C. Hair cortisol concentrations in war-affected adolescents: A prospective intervention trial. Psychoneuroendocrinology 89, 138-146 (2018).

63. Heim, C., Ehlert, U. \& Hellhammer, D. H. The potential role of hypocortisolism in the pathophysiology of stress-related bodily disorders. Psychoneuroendocrinology 25, 1-35 (2000).

64. Raffington, L., Schmiedek, F., Heim, C. \& Shing, Y. L. Cognitive control moderates parenting stress effects on children's diurnal cortisol. PLoS one 13, e0191215 (2018).

65. White, L. O. et al. Reduced hair cortisol after maltreatment mediates externalizing symptoms in middle childhood and adolescence. J. Child. Psychol. Psychiatry 58, 998-1007 (2017).

66. Saaltink, D.-J. \& Vreugdenhil, E. Stress, glucocorticoid receptors, and adult neurogenesis: a balance between excitation and inhibition? Cell. Mol. Life Sci. 71, 2499-2515 (2014)

67. Bryson, H. E., Goldfeld, S., Price, A. M. H. \& Mensah, F. Hair cortisol as a measure of the stress response to social adversity in young children. Developmental Psychobiology $\mathbf{0}$.

68. Malanchini, M. et al. Weak and uneven associations of home, neighborhood and school environments with stress hormone output across multiple time scales. bioRxiv 705996 (2019) https://doi.org/10.1101/705996.

69. Khoury, J. E., Enlow, M. B., Plamondon, A. \& Lyons-Ruth, K. The association between adversity and hair cortisol levels in humans: A meta-analysis. Psychoneuroendocrinology 103, 104-117 (2019).

70. Hellhammer, J. The Trier Social Stress Test (TSST) - a valid tool for clinical studies. Eur. J. Pharmacol. 668, e45-e46 (2011).

71. Wiedenmayer, C. P. et al. Cortisol levels and hippocampus volumes in healthy preadolescent children. Biol. Psychiatry 60, 856-861 (2006).

72. Gao, W. et al. Quantitative analysis of steroid hormones in human hair using a column-switching LC-APCI-MS/MS assay. J. Chromatogr. B 928, 1-8 (2013).

73. Carlitz, E. H. D., Kirschbaum, C., Stalder, T. \& van Schaik, C. P. Hair as a long-term retrospective cortisol calendar in orang-utans (Pongo spp.): New perspectives for stress monitoring in captive management and conservation. Gen. Comp. Endocrinol. 195, 151-156 (2014)

74. Burgess, N., Maguire, E. A. \& O’Keefe, J. The Human Hippocampus and Spatial and Episodic Memory. Neuron 35, 625-641 (2002).

75. Berron, D. et al. Strong Evidence for Pattern Separation in Human Dentate Gyrus. J. Neurosci. 36, 7569-7579 (2016).

76. Bender, A. R. et al. Optimization and validation of automated hippocampal subfield segmentation across the lifespan. Hum. Brain Mapp. 39, 916-931 (2018).

77. Yushkevich, P. A. et al. Automated volumetry and regional thickness analysis of hippocampal subfields and medial temporal cortical structures in mild cognitive impairment. Hum. Brain Mapp. 36, 258-287 (2015). 
78. Wisse, L. E. et al. A harmonized segmentation protocol for hippocampal and parahippocampal subregions: Why do we need one and what are the key goals? Hippocampus 27, 3-11 (2017).

79. Jack, C. R. et al. Anterior temporal lobes and hippocampal formations: normative volumetric measurements from MR images in young adults. Radiology 172, 549-554 (1989).

80. Raz, N. et al. Regional brain changes in aging healthy adults: general trends, individual differences and modifiers. Cereb. cortex 15 , 1676-1689 (2005).

81. Wennig, R. Potential problems with the interpretation of hair analysis results. Forensic Sci. Int. 107, 5-12 (2000).

82. Stalder, T. et al. Assessment of the cortisol awakening response: Expert consensus guidelines. Psychoneuroendocrinology 63, 414-432 (2016).

83. Abidin, R. R. Parenting Stress Index (PSI). (Pediatric Psychology Press Charlottesville, VA, 1990).

84. Tröster, H. Eltern-Belastungs-Inventar: EBI; deutsche Version des Parenting Stress Index (PSI) von RR Abidin. (Hogrefe, 2011).

85. Kessels, R. P., Hobbel, D. \& Postma, A. Aging, context memory and binding: A comparison of "what, where and when" in young and older adults. Int. J. Neurosci. 117, 795-810 (2007)

86. Kirwan, C. B. \& Stark, C. E. L. Overcoming interference: An fMRI investigation of pattern separation in the medial temporal lobe. Learn. Mem. 14, 625-633 (2007).

87. von Oertzen, T., Brandmaier, A. M. \& Tsang, S. Structural equation modeling with $\Omega$ nyx. Struct. Equ. Modeling: A Multidiscip. J. 22, 148-161 (2015).

88. Rosseel, Y. lavaan: An R Package for Structural Equation Modeling. J. Stat. Softw. 48, 1-36 (2012).

89. Riggins, T. et al. Protracted hippocampal development is associated with age-related improvements in memory during early childhood. NeuroImage 174, 127-137 (2018).

90. Schlichting, M. L. \& Preston, A. R. The hippocampus and memory integration: building knowledge to navigate future decisions. in The Hippocampus from Cells to Systems 405-437 (Springer, Cham, 2017). https://doi.org/10.1007/978-3-319-50406-3_13.

91. Krogsrud, S. K. et al. Development of hippocampal subfield volumes from 4 to 22 years. Hum. Brain Mapp. 35, 5646-5657 (2014).

92. Tamnes, C. K. et al. Regional hippocampal volumes and development predict learning and memory. Developmental Neurosci. 36, 161-174 (2014).

93. Merz, E. C. et al. Socioeconomic Disparities in Chronic Physiologic Stress Are Associated with Brain Structure in Children. Biological Psychiatry (2019) https://doi.org/10.1016/j.biopsych.2019.05.024.

94. DeMaster, D., Pathman, T., Lee, J. K. \& Ghetti, S. Structural development of the hippocampus and episodic memory: developmental differences along the anterior/posterior axis. Cereb. Cortex 24, 3036-3045 (2014).

95. Riggins, T., Blankenship, S. L., Mulligan, E., Rice, K. \& Redcay, E. Developmental differences in relations between episodic memory and hippocampal subregion volume during early childhood. Child. Dev. 86, 1710-1718 (2015).

96. Lindenberger, U., Von Oertzen, T., Ghisletta, P. \& Hertzog, C. Cross-sectional age variance extraction: what's change got to do with it? Psychol. aging 26, 34-47 (2011).

97. Kievit, R. A., Frankenhuis, W. E., Waldorp, L. J. \& Borsboom, D. Simpson's paradox in psychological science: a practical guide. Front Psychol 4, (2013).

98. Ngo, C. T., Newcombe, N. S. \& Olson, I. R. The ontogeny of relational memory and pattern separation. Dev Sci (2017) https://doi. org $/ 10.1111 /$ desc. 12556

99. Bakker, A., Kirwan, C. B., Miller, M. \& Stark, C. E. L. Pattern separation in the human hippocampal CA3 and dentate gyrus. Sci. 319, 1640-1642 (2008).

100. D’Esposito, M. \& Postle, B. R. The cognitive neuroscience of working memory. Annu. Rev. Psychol. 66, 115-142 (2015).

101. Ngo, C., Horner, A. J., Newcombe, N. \& Olson, I. Development of holistic episodic recollection. https://osf.io/x42d8 (2019) https:// doi.org/10.31234/osf.io/x42d8.

102. Ngo, C., Lin, Y., Newcombe, N. \& Olson, I. Building up and wearing down episodic memory: Mnemonic discrimination and relational binding. Journal of Experimental Psychology: General (2019) https://doi.org/10.1037/xge0000583.

103. Weckesser, L. J. et al. The psychometric properties and temporal dynamics of subjective stress, retrospectively assessed by different informants and questionnaires, and hair cortisol concentrations. Sci. Rep. 9, 1098 (2019).

104. Groeneveld, M. G. et al. Children's hair cortisol as a biomarker of stress at school entry. Stress. 16, 711-715 (2013).

105. Braig, S., Stalder, T., Kirschbaum, C., Rothenbacher, D. \& Genuneit, J. The association of potential stressors with hair steroids in parents with small children: The Ulm SPATZ health study. Psychoneuroendocrinology 102, 37-43 (2019).

106. Hamel, A. F. et al. Effects of shampoo and water washing on hair cortisol concentrations. Clinica Chim. Acta 412, 382-385 (2011).

107. Dettenborn, L., Tietze, A., Kirschbaum, C. \& Stalder, T. The assessment of cortisol in human hair: Associations with sociodemographic variables and potential confounders. Stress. 15, 578-588 (2012).

108. Serwinski, B., Salavecz, G., Kirschbaum, C. \& Steptoe, A. Associations between hair cortisol concentration, income, income dynamics and status incongruity in healthy middle-aged women. Psychoneuroendocrinology 67, 182-188 (2016).

109. Yushkevich, P. A. et al. User-guided 3D active contour segmentation of anatomical structures: significantly improved efficiency and reliability. Neuroimage 31, 1116-1128 (2006).

\section{Acknowledgements}

This work was supported by the Jacobs Foundation [grant 2014-1151 to YLS and CH] and conducted at the Center for Lifespan Psychology, Max Planck Institute for Human Development. During analyses and writing the manuscript, A.K. was supported by the Hungarian National Research, Development and Innovation Office NKFIH (FK 128648). The work of YLS was funded by a Minerva Research Group by the Max Planck Society, the European Union ((ERC-2018-StG-PIVOTAL-758898), and a Fellowship from the Jacobs Foundation (JRF 2018-2020). We thank all members of the Jacobs study team for their vital contribution, and all participants and family members for taking part in the study.

\section{Author contributions}

A.K., L.R., A.R.B., C.H., and Y.L.S. Contributed to the conception and design of the work, A.K., L.R., and K.B. acquired the data, A.K., L.R., A.R.B., K.B., and Y.L.S. performed the analyses, A.K., L.R., A.R.B., C.H., and Y.L.S. contributed to the interpretation of the data, A.K. drafted the manuscript, and A.K., L.R., A.R.B., K.B., C.H., and Y.L.S. revised it.

\section{Competing interests}

The authors declare no competing interests. 


\section{Additional information}

Supplementary information is available for this paper at https://doi.org/10.1038/s41598-020-61131-x.

Correspondence and requests for materials should be addressed to A.K. or C.H.

Reprints and permissions information is available at www.nature.com/reprints.

Publisher's note Springer Nature remains neutral with regard to jurisdictional claims in published maps and institutional affiliations.

(c) (i) Open Access This article is licensed under a Creative Commons Attribution 4.0 International License, which permits use, sharing, adaptation, distribution and reproduction in any medium or format, as long as you give appropriate credit to the original author(s) and the source, provide a link to the Creative Commons license, and indicate if changes were made. The images or other third party material in this article are included in the article's Creative Commons license, unless indicated otherwise in a credit line to the material. If material is not included in the article's Creative Commons license and your intended use is not permitted by statutory regulation or exceeds the permitted use, you will need to obtain permission directly from the copyright holder. To view a copy of this license, visit http://creativecommons.org/licenses/by/4.0/.

(C) The Author(s) 2020 